\title{
ARTICLE OPEN \\ Visualizing ferromagnetic domain behavior of magnetic topological insulator thin films
}

\author{
Wenbo Wang ${ }^{1}$, Cui-Zu Chang ${ }^{2}$, Jagadeesh S Moodera ${ }^{2}$ and Weida Wu ${ }^{1}$
}

A systematic magnetic force microscopy (MFM) study of domain behavior in thin films of the magnetic topological insulator $\mathrm{Sb}_{1.89} \mathrm{~V}_{0.11} \mathrm{Te}_{3}$ reveals that in the virgin domain state, after zero-field cooling, an equal population of up and down domains occurs. Interestingly, the cooling field dependence of MFM images demonstrates that a small cooling magnetic field (approximately 5-10 Oe) is sufficient to significantly polarize the film despite the coercive field $\left(H_{\mathrm{C}}\right)$ for these films being on the order of a tesla at low temperature. By visualizing the magnetization reversal process around $H_{C}$ of $\mathrm{V}$-doped $\mathrm{Sb}_{2} \mathrm{Te}_{3}$, we observed a typical domain behavior of a ferromagnet, i.e., domain nucleation and domain wall propagation. Our results provide direct evidence of ferromagnetic behavior of the magnetic topological insulator, a necessary condition for a robust quantum anomalous Hall effect.

npj Quantum Materials (2016) 1, 16023; doi:10.1038/npjquantmats.2016.23; published online 21 October 2016

\section{INTRODUCTION}

The quantum anomalous Hall effect (QAHE), i.e., quantum Hall effect (QHE) without external magnetic field, is of great fundamental interest and of potential application in metrology. ${ }^{1-10}$ Similar to QHE, the microscopic mechanism of QAHE is due to the presence of dissipationless chiral edge states. The realization of QAHE requires two criteria: the breaking of time reversal symmetry and topologically nontrivial band structure. In principle, there are several ways to realize the $\mathrm{QAH}$ state. So far, introducing ferromagnetism into a topological insulator (TI) is the only viable route to realize the fascinating $\mathrm{QAH}$ state. The ferromagnetic behavior has been observed by doping Tls with various transition metal elements, such as $\mathrm{Cr}, \mathrm{V}$, and $\mathrm{Mn} .{ }^{11-17}$ Yet, it is challenging to control the Fermi level in the exchange gap region in these materials so that the transport is dominated by the chiral edge states for observing clear quantized Hall conductance. The breakthrough was accomplished by Chang et al., who demonstrated QAHE (at $30 \mathrm{mK}$ ) in $\mathrm{Cr}$-doped $\mathrm{Bi}_{x} \mathrm{Sb}_{2-x} \mathrm{Te}_{3}$ (BST) thin films synthesized by molecular beam epitaxy, ${ }^{18}$ soon confirmed by several other groups. ${ }^{19-22}$ However, the QAH state is very fragile in the $\mathrm{Cr}$-doped BST thin films, where quantized Hall conductance was observed at $\mathrm{mK}$ temperature. It was shown that modulation doping of $\mathrm{Cr}$ can enhance QAH temperature. ${ }^{23}$ On the other hand, a high precision robust QAH state was observed in V-doped BST thin films ${ }^{24}$ at a higher temperature $(\sim 130 \mathrm{mK})$ with the same doping level. V-doped BST is a hard ferromagnet with a larger coercive field $\left(H_{C} \sim 1.0 \mathrm{~T}\right)$ and higher Curie temperature $\left(T_{C}\right){ }^{24}$ These features make the V-doped BST thin films, if combined with modulation doping, more preferable to realizing QAHE at higher temperature.

In addition to the robust QAH effect, a remarkable spontaneous self-magnetization effect has been reported in V-doped BST thin films. ${ }^{24}$ The non-zero net magnetization develops in the virgin state without the assistance of external magnetic fields, which is not commonly seen in conventional ferromagnetic materials with some exceptions in soft magnetic thin films. ${ }^{25,26}$ In contrast, slow relaxation behaviors were observed in transport studies, indicating superparamagnetism due to inhomogeneity. ${ }^{27,28}$ The superparamagnetic dynamic behavior of the quantum phase transition between the two anomalous Hall plateaus was visualized in Cr-doped BST thin films using scanning nanoSQUID (nano superconducting quantum interference device). ${ }^{29}$ However, recent transport measurements at lower temperatures revealed large discrete jumps near the coercive field, suggesting domain-like behavior. ${ }^{30}$ It is not clear whether this superparamagnetic behavior is universally favorable in other magnetic TI thin films such as V-doped BST. In order to resolve these mysteries, we utilized magnetic force microscopy (MFM) technique to investigate the domain behavior of $\mathrm{V}$-doped $\mathrm{Sb}_{2} \mathrm{Te}_{3}$ thin films.

In this Article, we report cryogenic MFM studies of the domain states of $\mathrm{Sb}_{1.89} \mathrm{~V}_{0.11} \mathrm{Te}_{3}$ thin films. A zero magnetization state with equal population of up and down domains was observed after zero field cooling (ZFC). Our MFM results reveal that a small external magnetic field (approximately 5-10 Oe) is sufficient to polarize the magnetization of the film, which provides a physical explanation of the 'self-magnetization' mystery. ${ }^{24}$ The observed domain states are consistent with in situ magnetoresistance measurements, suggesting that our MFM observation is representative of the bulk properties. In contrast to the superparamagnetic behavior in previous reports of $\mathrm{Cr}$-doped BST, our MFM results reveal a typical ferromagnetic domain behavior of the magnetization reversal process via nucleation and domain wall propagation in V-doped $\mathrm{Sb}_{2} \mathrm{Te}_{3}$ thin films. Since the ferromagnetism in V doped BST varies little with Bi doping, our MFM results provide supporting evidence of the correlation between improved ferromagnetism and enhanced QAHE. ${ }^{24}$

\section{RESULTS AND DISCUSSION}

Figure 1 shows topographic and MFM images of the $\mathrm{Sb}_{1.89} \mathrm{~V}_{0.11} \mathrm{Te}_{3}$ thin film at $5 \mathrm{~K}$ after ZFC and field cooling (FC). The topographic image shows a flat surface with roughness $\sim 1.9 \mathrm{~nm}$. The topographic features are likely surface contamination after the film was

\footnotetext{
${ }^{1}$ Department of Physics and Astronomy, Rutgers University, Piscataway, NJ, USA and ${ }^{2}$ Francis Bitter Magnet Lab, Massachusetts Institute of Technology, Cambridge, MA, USA. Correspondence: W Wu (wdwu@physics.rutgers.edu)

Received 25 July 2016; revised 9 September 2016; accepted 13 September 2016
} 

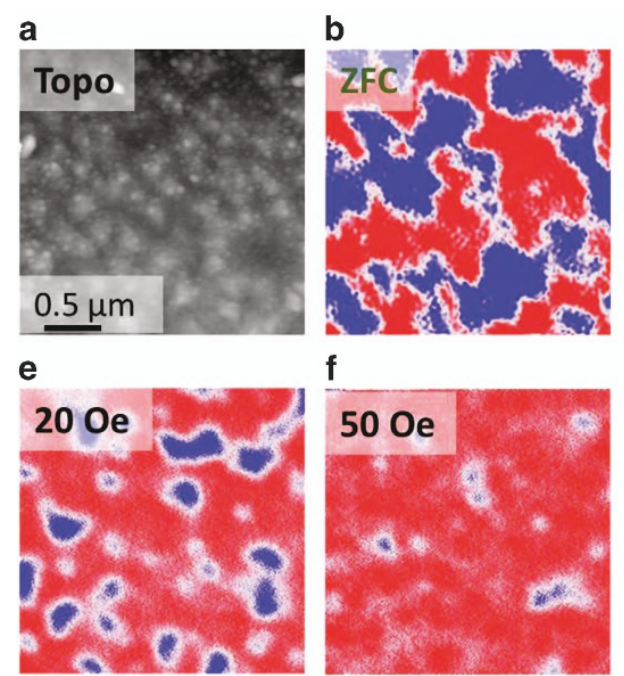

g

f
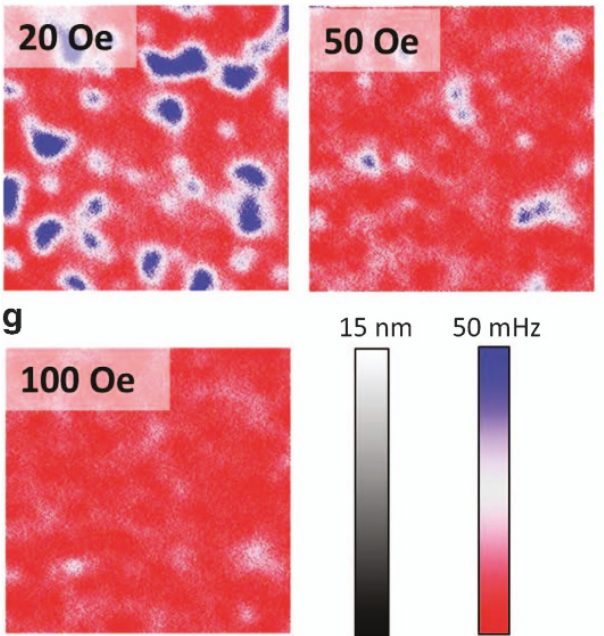

$15 \mathrm{~nm} \quad 50 \mathrm{mHz}$

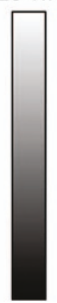

c

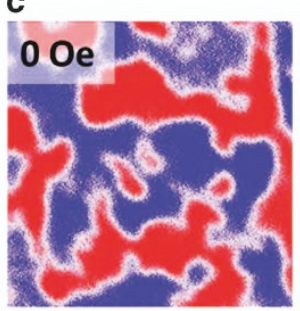

h

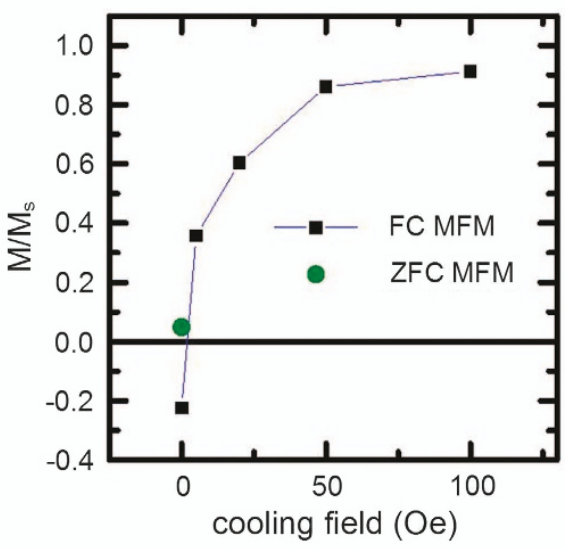

Figure 1. Topographic (a) and MFM image (b) measured at $5 \mathrm{~K}$ after ZFC. The equal population of up (red) and down (blue) domains indicates a zero-magnetization virgin domain state. $(\mathbf{c}-\mathbf{g})$ MFM images $(5 \mathrm{~K})$ of FC domain states with cooling fields ranging from 0 to 100 Oe. The cooling field values are labeled at the upper-left corner of each MFM image. The color scale is $15 \mathrm{~nm}(50 \mathrm{mHz})$ for topographic (MFM) image(s), and $20 \mathrm{mHz}$ for the ZFC MFM image (b). Note that the ZFC image (b) was slightly filtered to enhance the signal to noise ratio. (See raw data in Supplementary Figure S1). (h) Cooling field dependence of the net magnetization of various domain states is inferred from MFM images using a histogram analysis of MFM images. The trapped flux in the superconducting magnet is estimated to be $\sim 3$ Oe.

exposed to air. The virgin state after ZFC, as shown in Figure $1 \mathrm{~b}$, exhibits a multi-domain state which is unlike the reported selfmagnetization behavior. Figures $1 \mathrm{c}-\mathrm{g}$ show MFM images of the same region at $5 \mathrm{~K}$ after $\mathrm{FC}$. The sample was thermally cycled to $60 \mathrm{~K}$, then cooled down to $5 \mathrm{~K}$ with the superconducting magnet set to the field values labeled on the upper left corner of each panel in Figure 1. As shown in Figure 1c, the nominal 0 Oe FC data shows that the sample is slightly negatively polarized, indicating the existence of negative trapped flux in the superconducting magnet. This is consistent with the history of the superconducting magnet usage, since the field was ramped down from a positive value to zero, i.e., negative trapped flux due to a slight overshot of the power supply. Applying a small field (e.g., 5 Oe) during cooling can significantly polarize the film (see Figure 1d) and the film saturates at 100 Oe (see Figure 1g), indicating divergent correlation length at $T_{\mathrm{C}}$ due to strong ferromagnetic exchange interactions. The systematic variation of domain population is a strong evidence of long-range ferromagnetic order in V-doped $\mathrm{Sb}_{2} \mathrm{Te}_{3}$.

In order to quantitatively characterize the domain states after different field cooling, the population of positive and negative domains was estimated by conducting a histogram analysis of these MFM images. The histogram curves exhibit two peaks which correspond to up and down domains (Supplementary Figure S2). We used the peak height to estimate the population of up and down domains. The normalized magnetization of the thin film $M / M_{S}\left(M_{s}\right.$ : saturation magnetization) therefore can be estimated from: $\left(N_{\uparrow}-N_{\downarrow}\right) /\left(N_{\uparrow}+N_{\downarrow}\right)$, where $N_{\uparrow}$ and $N_{\downarrow}$ are populations of up and down domains, respectively. The normalized magnetization as a function of cooling field is plotted in Figure $1 \mathrm{~h}$. The trapped flux of our superconducting magnet inferred from linear extrapolation is $\sim 3$ Oe. Therefore, the previously observed selfmagnetization effect is likely an extrinsic effect due to non-zero trapped flux in the superconducting magnet. ${ }^{24}$

The ferromagnetic domain behavior was also investigated by performing field-dependent MFM imaging of the domain state after ZFC, as shown in Figure 2. The ZFC domain state is essentially unchanged up to $0.4 \mathrm{~T}$, indicating a strong domain wall pinning effect of a hard ferromagnet. ${ }^{24}$ The domain contrast enhancement between 0 and $0.4 \mathrm{~T}$ is due to the enhancement of the MFM tip moment by the external magnetic field. Higher magnetic fields induce more red regions (up domains), where spins are parallel with external fields. The film reaches saturation at $1.5 \mathrm{~T}$, as no blue region (down domain) remains. There are some weak MFM contrasts in the saturation state, which anti-correlate with topographic features shown in Figure 1a. So they are nonmagnetic features, possibly originating from residual electrostatic interactions due to the variation of contact potential caused by surface contamination. The film remained in the saturation state after the field was ramped down to zero, suggesting a strong uniaxial anisotropy that prevents domain nucleation at zero field. This is in sharp contrast with the superparamagnetic behavior observed in prior studies of Cr-doped BST, where substantial relaxation was observed at zero and low fields. ${ }^{29}$ For our sample, significant relaxation was observed near the coercive field.

The down domains started to nucleate at $-0.5 \mathrm{~T}$. Further decrease in $H$ induced more down domains (blue region). A fifty-fifty domain state was recovered at approximately -0.7 to $-0.8 \mathrm{~T}$, which is defined as the coercive field $\left(H_{C}\right)$. Significant domain relaxation was observed near $H_{C}$ as shown by sharp domain 
a

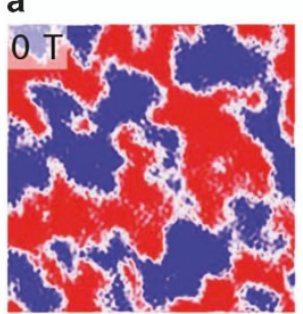

e

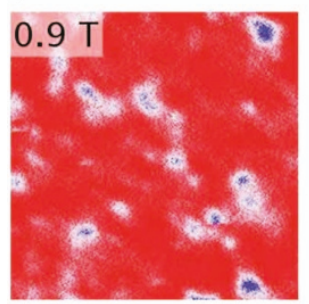

i

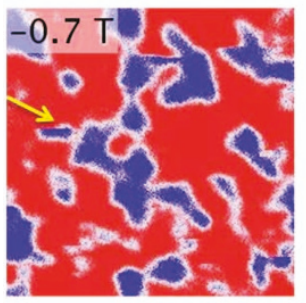

k

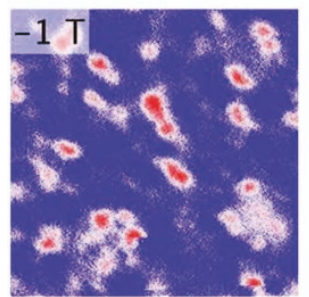

b

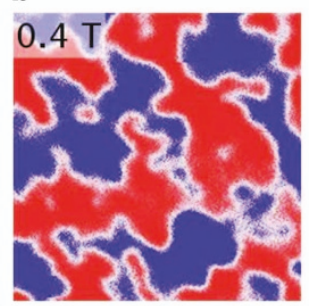

f

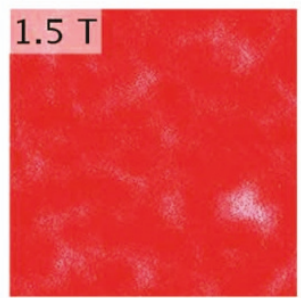

j

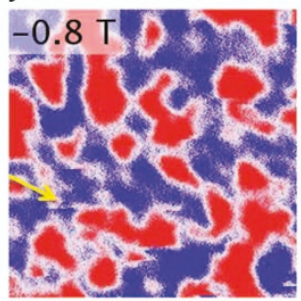

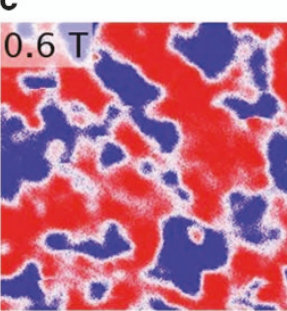

g

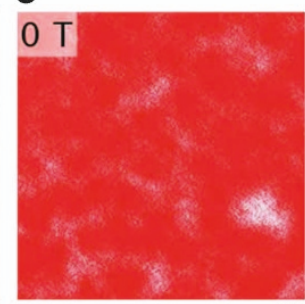

d

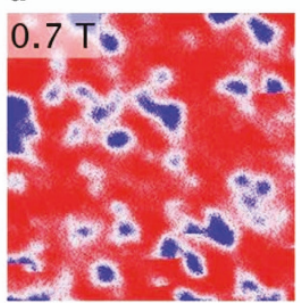

h

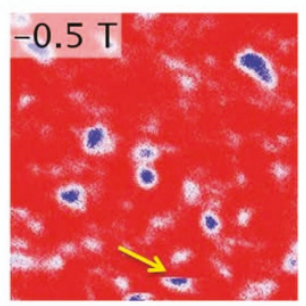

m

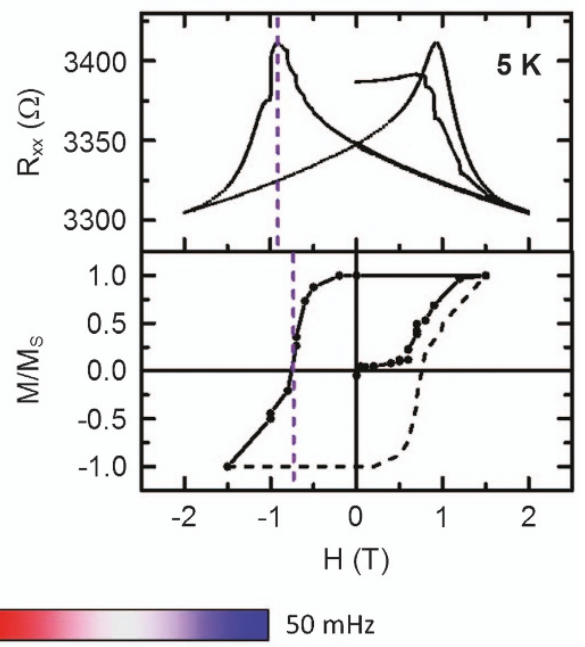

Figure 2. (a-I) $5 \mathrm{~K}$ field-dependent MFM images of the virgin domain state after ZFC show a typical domain behavior with a strong pinning effect. The applied magnetic fields are labeled at the top-left corner of each MFM image. (f) The field dependence of 2-probe resistance $R_{x x}$ was measured with the field sweeping within $\pm 2 \mathrm{~T}$. The $\mathrm{M}-\mathrm{H}$ hysteresis loop deduced from MFM images is qualitatively consistent with the R$\mathrm{H}$ butterfly loop. The coercive field is $0.92 \mathrm{~T}$ from $\mathrm{R}-\mathrm{H}$ and $0.76 \mathrm{~T}$ from $\mathrm{M}-\mathrm{H}$.

switching along the slow scan axis in MFM images near coercive fields, e.g., as indicated by arrows in Figures $2 \mathrm{~h}-\mathrm{j}$. At $-1.5 \mathrm{~T}$, the sample was fully polarized (Figure 2l). The magnetization reversal process observed in the $10 \mathrm{QL} \mathrm{Sb}{ }_{1.89} \mathrm{~V}_{0.11} \mathrm{Te}_{3}$ thin film is more consistent with typical ferromagnetic domain reversal behavior, including domain nucleation and domain wall propagation. ${ }^{26}$ The $M(H)$ hysteresis loop deduced from MFM images is plotted in Figure $2 \mathrm{~m}$. Note that we only measured the virgin curve and half of the hysteresis loop. The other half (dashed line) was replicated from measured results via a symmetric operation, assuming the hysteresis loop is symmetric about zero field. The coercive field $\left(H_{\mathrm{C}}\right)$ deduced from MFM images is $\sim 0.76 \mathrm{~T}$.

The in situ two-probe resistance $(R)$ measurement was performed simultaneously with MFM measurement. The $R(H)$ butterfly loop is qualitatively consistent with the $M(H)$ loop (Figure $2 \mathrm{~m}$ ) though the $H_{C}$ from transport $(\sim 0.92 \mathrm{~T})$ is slightly higher. The difference likely originates from the stray field from the MFM tip, which locally adds to the external magnetic field. ${ }^{17}$ So the scanned region (probed by MFM) experiences a higher magnetic field than the rest of the sample (probed by $R$ ). Note that it took some time (approximately 5-10 $\mathrm{min}$ ) to capture each MFM image. The steps in the $R(H)$ loop indicate a substantial time relaxation effect around the coercive field. Although similar relaxation behavior was also observed in $\mathrm{Cr}$-doped magnetic $\mathrm{Tls}^{27-29}$ the relaxation in V-doped films is more consistent with typical ferromagnetic domain behavior, i.e., domain nucleation and domain wall propagation (Supplementary Figure S3). It is interesting to note that a recent transport study at ultralow temperature $(10-200 \mathrm{mK})$ also revealed domain-like behavior in $\mathrm{Cr}$ doped $\mathrm{BST}^{30}$ indicating much softer ferromagnetism in Cr-doped systems.

Further evidence of ferromagnetic domain behavior comes from the robust domain pattern against thermal fluctuation at elevated temperature up to $20 \mathrm{~K}$ (Supplementary Figure S4). The domain contrast quickly diminished upon warming the film to $35 \mathrm{~K}$. This is consistent with $T_{C} \sim 32 \mathrm{~K}$ deduced from $R(T)$ data (Supplementary Figure S4). ${ }^{17}$ The agreement between bulk transport measurement and local MFM measurement suggests that our MFM results reflect a representative domain behavior of the $\mathrm{Sb}_{1.89} \mathrm{~V}_{0.11} \mathrm{Te}_{3}$ thin film. Although $\mathrm{V}$ doped $\mathrm{Sb}_{2} \mathrm{Te}_{3}$ is not insulating because of substantial bulk carrier concentration, introducing a small amount of $\mathrm{Bi}$ can tune the Fermi level to 
a

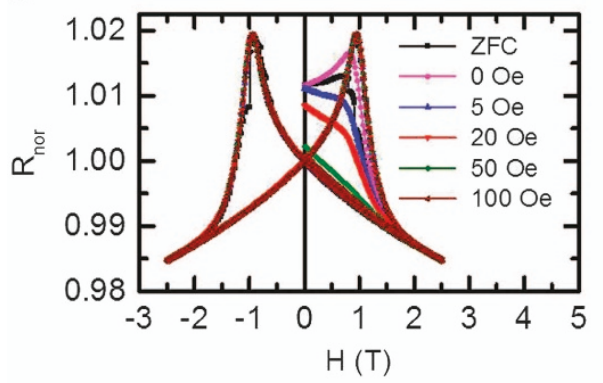

b

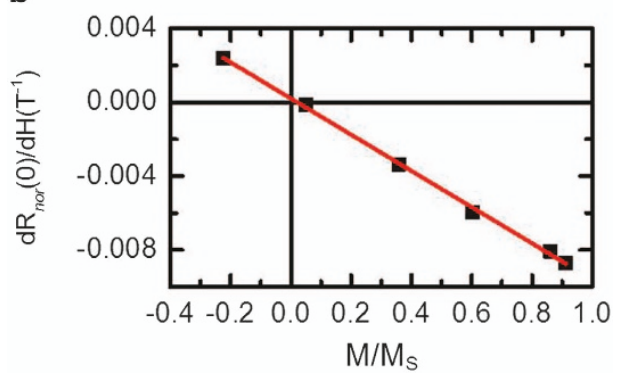

Figure 3. (a) Field dependence of normalized resistance $R_{\text {nor }}=R_{x x}(H) / R_{x x}(0)$. Magnetoresistance $\left(R_{x x}\right)$ was measured with the magnetic field sweeping within $\pm 2.5 \mathrm{~T}$ after FC (0 to $100 \mathrm{Oe}$ ). (b) Graph of the initial slope of normalized resistance versus normalized net magnetization. The linear relationship between them is evidenced from the linear fit (red line).

the Dirac point. ${ }^{24}$ Since the ferromagnetism $\left(T_{C}\right.$ and $\left.H_{C}\right)$ of $\mathrm{V}$ doped BST weakly depends on the concentration of $\mathrm{Bi}^{24}$ we expect similar ferromagnetic domain behavior in V-doped BST that shows QAHE at low temperature.

Figure 3 shows in situ magnetoresistance (MR) measurement of different FC domain states. The initial MR curves of different domain states show a systematic behavior. First, the single domain state is more conducting than the multidomain state, as the zerofield resistance $R(0)$ of 100 Oe FC is significantly less than ZFC, while $R(0)$ of 0 and 5 Oe FC are very close to ZFC. The difference may be related to the density of domain walls in different domain states. The more polarized film has smaller domain wall density. It is known that the domain walls enhance electron scattering in ferromagnetic metals, ${ }^{31,32}$ thus, the multi-domain state is usually more resistive. Second, the slope of the MR curve at zero field is proportional to the net magnetization, as shown in Figure $3 \mathrm{~b}$. This can be understood in terms of averaged MR of the multi-domain state. For domains with magnetization along the direction of the external magnetic field, the intensity of thermal excitation, i.e., spin wave excitation (magnon), will be suppressed by the external field. Therefore magnon-electron scattering will be suppressed, and thus resistivity will be lowered. Conversely, the magnonelectron scattering will be enhanced for domains with magnetization opposite to the direction of the external magnetic field because of enhancement of spin wave intensity. Assuming a linear response at the zero-field limit, the change in resistance due to the external magnetic field for positive or negative domains is $\Delta R_{ \pm}=\mp k \cdot H(k>0)$. Assuming the fraction of up (down) domains is $N_{\uparrow}\left(N_{\downarrow}\right)$ and a simple linear resistance model, the net resistance change would be, $\Delta R=N_{\uparrow} \cdot \Delta R_{\uparrow}+N_{\downarrow} \cdot \Delta R_{\downarrow}=k \cdot\left(N_{\downarrow}-N_{\uparrow}\right) \cdot H$, i.e., $\left.\frac{\mathrm{d} R}{\mathrm{~d} H}\right|_{H=0}=k\left(N_{\downarrow}-N_{\uparrow}\right) \propto-M / M_{\mathrm{S}}$, in good agreement with results in Figure $3 \mathrm{~b}$. Note that the transport properties of $\mathrm{V}$-doped $\mathrm{Sb}_{2} \mathrm{Te}_{3}$ are dominated by bulk (valence band) carriers. ${ }^{24}$ Such MR behavior due to bulk carriers would diminish in insulating $\mathrm{V}$ doped BST at low temperature where QAH chiral edge states dominate the transport.

\section{CONCLUSION}

In conclusion, we present a systematic characterization of ferromagnetic domain behavior of $\mathrm{Sb}_{1.89} \mathrm{~V}_{0.11} \mathrm{Te}_{3}$ molecular beam epitaxy thin film using cryogenic MFM. A zero net magnetization domain state was observed in $10 \mathrm{QL} \mathrm{Sb}{ }_{1.89} \mathrm{~V}_{0.11} \mathrm{Te}_{3}$ thin film when cooled in zero $H$. The magnetization reversal process at $H_{C}$ shows typical domain behavior of a hard ferromagnet with strong out-ofplane uniaxial anisotropy. In contrast to $\mathrm{Cr}$-doped TI thin films, which exhibit strong inhomogeneity and superparamagnetic behavior of nano-islands, ${ }^{29}$ the V-doped magnetic TI thin films are more homogeneous with less disorder. Furthermore, the film can be significantly polarized with a small field (approximately 5-10 Oe) applied during cooling through $T_{C}$, which explains the 'self-magnetization' behaviors in the previous report. ${ }^{24}$ Compared with Cr-doped Tls, the V-doped magnetic Tls are more stable in the single domain state due to their high anisotropy and coercivity as well as lower magnetization. ${ }^{24}$ This property helps V-doped magnetic $\mathrm{TI}$ films enter the single domain state at relatively higher temperature with the presence of a small magnetic field (e.g., trapped flux in a superconducting magnet, earth field, and so on), which is a necessary condition for observing QAH effect. Our MFM studies of V-doped magnetic TI thin films shed light on the path to high-temperature QAH materials.

\section{MATERIALS AND METHODS}

\section{Sample preparation}

Epitaxial thin film $\mathrm{Sb}_{1.89} \mathrm{~V}_{0.11} \mathrm{Te}_{3}$ capped with $10 \mathrm{~nm}$ Te was grown on heat treated $\mathrm{SrTiO}_{3}(111)$ substrate by co-evaporation in a molecular beam epitaxy system. The nominal thickness of the film is 10 quintuple layers (QL).

\section{MFM measurement}

The MFM experiments were carried out in a homemade cryogenic atomic force microscope (AFM) using commercial piezoresistive cantilevers (spring constant $k \approx 3 \mathrm{~N} / \mathrm{m}$, resonant frequency $f_{0} \approx 42 \mathrm{kHz}$ ). The homemade AFM is interfaced with a Nanonis SPM Controller (SPECS) and a commercial phaselock loop (SPECS). ${ }^{17}$ MFM tips were prepared by depositing nominally $100 \mathrm{~nm}$ Co film onto bare tips using e-beam evaporation. MFM images were taken in a constant mode with the scanning plane $\sim 40 \mathrm{~nm}$ above the sample surface. MFM signal, the change of cantilever resonant frequency, is proportional to out-of-plane stray field gradient. ${ }^{33}$ Electrostatic interaction was minimized by nulling the tip-surface contact potential difference. Red (blue) regions in MFM images represent up (down) ferromagnetic domains, where magnetizations are parallel (anti-parallel) with the positive external field.

\section{MR measurement}

In-situ 2-probe resistance of the thin-film samples was measured with a digital multimeter (Keithley 2100).

\section{ZFC experiments}

ZFC MFM measurements were carried out after warming the superconducting magnet to room temperature to eliminate trapped magnetic flux. During ZFC, the MFM tip was $>100 \mu \mathrm{m}$ above sample surface to minimize tip stray magnetic field on the surface.

\section{ACKNOWLEDGEMENTS}

This work at Rutgers is supported by the Office of Basic Energy Sciences, Division of Materials Sciences and Engineering, U.S. Department of Energy under Award number DE-SC0008147. The work at MIT is supported by grants NSF (DMR-1207469), NSF (DMR-0819762) (MIT MRSEC), ONR (N00014-13-1-0301), and the STC Center for Integrated Quantum Materials under NSF grant DMR-1231319. 


\section{CONTRIBUTIONS}

W. B. W. and W. D. W. conceived and designed the research. C.-Z. C. grew the samples. W. B. W. performed the MFM and in situ transport measurements. W. B. W. analyzed the data and wrote the manuscript with contributions from all authors.

\section{COMPETING INTERESTS}

The authors declare no conflict of interest.

\section{REFERENCES}

1. Haldane, F. D. M. Model for a quantum hall effect without landau levels: Condensed-matter realization of the 'parity anomaly'. Phys. Rev. Lett. 61, 2015-2018 (1988).

2. Onoda, M. \& Nagaosa, N. Quantized anomalous Hall effect in two-dimensional ferromagnets: Quantum Hall effect in metals. Phys. Rev. Lett. 90, 206601 (2003).

3. Liu, C. X., Qi, X. L., Dai, X., Fang, Z. \& Zhang, S. C. Quantum anomalous Hall effect in $\mathrm{Hg}_{1-y} \mathrm{Mn}_{y} \mathrm{Te}$ quantum wells. Phys. Rev. Lett. 101, 146802 (2008).

4. Qiao, Z. et al. Quantum anomalous Hall effect in graphene proximity coupled to an antiferromagnetic insulator. Phys. Rev. Lett. 112, 116404 (2014).

5. Qiao, Z. H. et al. Quantum anomalous Hall effect in graphene from Rashba and exchange effects. Phys. Rev. B 82, 161414 (2010)

6. Zhang, H., Lazo, C., Bluegel, S., Heinze, S. \& Mokrousov, Y. Electrically tunable quantum anomalous Hall effect in graphene decorated by $5 \mathrm{~d}$ transition-metal adatoms. Phys. Rev. Lett. 108, 056802 (2012)

7. Ezawa, M. Valley-polarized metals and quantum anomalous Hall effect in silicene. Phys. Rev. Lett. 109, 055502 (2012).

8. Nomura, K. \& Nagaosa, N. Surface-quantized anomalous Hall current and the magnetoelectric effect in magnetically disordered topological insulators. Phys. Rev. Lett. 106, 166802 (2011).

9. Yu, R. et al. Quantized anomalous Hall effect in magnetic topological insulators. Science 329, 61-64 (2010).

10. Qi, X. L., Hughes, T. L. \& Zhang, S. C. Topological field theory of time-reversal invariant insulators. Phys. Rev. B 78, 195424 (2008).

11. Hor, Y. S. et al. Development of ferromagnetism in the doped topological insulator $\mathrm{Bi}_{2-x} \mathrm{Mn}_{x} \mathrm{Te}_{3}$. Phys. Rev. B 81, 195203 (2010).

12. Dyck, J. S., Drasar, C., Lost'ak, P. \& Uher, C. Low-temperature ferromagnetic properties of the diluted magnetic semiconductor $\mathrm{Sb}_{2-x} \mathrm{Cr}_{x} \mathrm{Te}_{3}$. Phys. Rev. B 71 115214 (2005)

13. Dyck, J. S., Hajek, P., Losit'ak, P. \& Uher, C. Diluted magnetic semiconductors based on $\mathrm{Sb}_{2-x} \mathrm{~V}_{x} \mathrm{Te}_{3}(0.01 \leq \mathrm{x} \leq 0.03)$. Phys. Rev. B 65, 115212 (2002).

14. Zhou, Z., Chien, Y. J. \& Uher, C. Thin film dilute ferromagnetic semiconductors $\mathrm{Sb}_{2-x} \mathrm{Cr}_{x} \mathrm{Te} 3$ with a Curie temperature up to 190 K. Phys. Rev. B 74, 224418 (2006).

15. Haazen, P. P. J. et al. Ferromagnetism in thin-film Cr-doped topological insulator $\mathrm{Bi}_{2} \mathrm{Se}_{3}$. Appl. Phys. Lett. 100, 082404 (2012).

16. $\mathrm{Li}, \mathrm{H}$. et al. Carriers dependence of the magnetic properties in magnetic topological insulator $\mathrm{Sb}_{1.95-x} \mathrm{Bi}_{x} \mathrm{Cr}_{0.05} \mathrm{Te}_{3}$. Appl. Phys. Lett. 101, 072406 (2012).
17. Wang, W. et al. Visualizing ferromagnetic domains in magnetic topological insulators. APL Mater. 3, 083301 (2015).

18. Chang, C. Z. et al. Experimental observation of the quantum anomalous Hall effect in a magnetic topological insulator. Science 340, 167-170 (2013).

19. Checkelsky, J. G. et al. Trajectory of the anomalous hall effect towards the quantized state in a ferromagnetic topological insulator. Nat. Phys. 10, 731-736 (2014).

20. Kou, X. et al. Scale-invariant quantum anomalous hall effect in magnetic topological insulators beyond the two-dimensional limit. Phys. Rev. Lett. 113, 137201 (2014)

21. Kou, X. et al. Metal-to-insulator switching in quantum anomalous Hall states. Nat. Commun. 6, 8474 (2015).

22. Feng, Y. et al. Observation of the zero Hall plateau in a quantum anomalous Hall insulator. Phys. Rev. Lett. 115, 126801 (2015).

23. Mogi, M. et al. Magnetic modulation doping in topological insulators toward higher-temperature quantum anomalous Hall effect. Appl. Phys. Lett. 107, 182401 (2015).

24. Chang, C. Z. et al. High-precision realization of robust quantum anomalous Hall state in a hard ferromagnetic topological insulator. Nat. Mater. 14, 473-477 (2015).

25. Hubert, A. \& Schafer, R. Magnetic Domains. Springer Berlin Heidelberg, (1998).

26. Schwarz, A. et al. Visualization of the Barkhausen effect by magnetic force microscopy. Phys. Rev. Lett. 92, 077206 (2004).

27. Bestwick, A. J. et al. Precise quantization of the anomalous Hall effect near zero magnetic field. Phys. Rev. Lett. 114, 187201 (2015).

28. Grauer, S. et al. Coincidence of superparamagnetism and perfect quantization in the quantum anomalous Hall state. Phys. Rev. B 92, 201304 (2015).

29. Lachman, E. O. et al. Visualization of superparamagnetic dynamics in magnetic topological insulators. Sci. Adv. 1, e1500740 (2015).

30. Liu, M. et al. Large discrete jumps observed in the transition between Chern states in a ferromagnetic topological insulator. Sci. Adv. 2, e1600167 (2016).

31. Gregg, J. F. et al. Giant magnetoresistive effects in a single element magnetic thin film. Phys. Rev. Lett. 77, 1580-1583 (1996).

32. Kent, A. D., Yu, J., Rudiger, U. \& Parkin, S. S. P. Domain wall resistivity in epitaxial thin film microstructures. J. Phys. Condens. Matter 13, R461-R488 (2001).

33. Rugar, D. et al. Magnetic force microscopy: general principles and application to longitudinal recording media. J. Appl. Phys. 68, 1169-1183 (1990).

(c) (i) This work is licensed under a Creative Commons Attribution 4.0 article are included in the article's Creative Commons license, unless indicated otherwise in the credit line; if the material is not included under the Creative Commons license, users will need to obtain permission from the license holder to reproduce the material. To view a copy of this license, visit http://creativecommons.org/licenses/ by/4.0/

(c) The Author(s) 2016

Supplementary Information accompanies the paper on the npj Quantum Materials website (http://www.nature.com/npjquantmats) 